\title{
PELÍCULAS COMESTÍVEIS EM FRUTAS CONSERVADAS POR MÉTODOS COMBINADOS: POTENCIAL DA APLICAÇÃO
}

\author{
HENRIETTE MONTEIRO CORDEIRO DE AZEREDO *
}

\begin{abstract}
Efetuou-se revisão de literatura sobre películas comestíveis e suas possíveis aplicações para melhorar a qualidade de frutas conservadas por métodos combinados. São abordadas a redução da perda de umidade das frutas durante a estocagem, aumento na relação de performance durante a desidratação osmótica, melhoria da aparência das frutas, redução do crescimento microbiano e retenção de compostos de aroma. As películas, além de aumentar a estabillidade dos produtos de frutas podem melhorar sua qualidade sensorial.
\end{abstract}

PALAVRAS-CHAVE: TECNOLOGIA DE MÉTODOS COMBINADOS; CONSERVAÇÃO DE FRUTAS; PELÍCULAS COMESTÍVEIS.

\section{INTRODUÇÃO}

A demanda dos consumidores por alimentos mais frescos e convenientes tem aumentado muito nas últimas décadas. Em razão disso, a tecnologia de métodos combinados (TMC), também conhecida como tecnologia de obstáculos (hurdle technology), tem sido muito visada pelos pesquisadores. Essa tecnologia tem como base a combinação de dois ou mais obstáculos ao crescimento microbiano, aplicados em baixos níveis, para promover a estabilidade do alimento em temperatura ambiente (ALZAMORA et al., 1993). A inibição ao crescimento microbiano, ao invés de ser alcançada pela aplicação drástica de um único fator de conservação, é obtida por efeito sinérgico da combinação de vários fatores ou obstáculos (LEISTNER, 1992). Com isso, os produtos obtidos por TMC são similares aos frescos, principalmente em termos de sabor e textura, apresentando, portanto, maior aceitação pelos consumidores.

* Engenheira de Alimentos, Doutora em Tecnologia de Alimentos, Embrapa Agroindústria Tropical, Fortaleza, CE (e-mail: ette@cnpat.embrapa.br). 
Entre os fatores de conservação, geralmente, utilizados em combinação, podem ser mencionados pequena redução na atividade de água, redução do pH e adição de conservantes químicos (CHIRIFE e FAVETTO, 1992; AGUILERA e CHIRIFE, 1994).

O objetivo deste trabalho de revisão foi reunir dados sobre possíveis aplicações de películas comestíveis em frutas conservadas por métodos combinados.

\section{PELÍCULAS COMESTÍVEIS}

As películas comestíveis podem ser classificadas em filmes e coberturas. Embora os termos sejam muitas vezes utilizados indiscriminadamente, a diferença básica é que os filmes são préformados, separadamente, do produto. Já as coberturas são formadas sobre a própria superfície do alimento, o que pode ser efetuado, por exemplo, por imersão ou aspersão (KESTER e FENNEMA, 1986).

As características requeridas da película comestível dependem, principalmente, das características do alimento. Assim, para produtos suscetíveis à oxidação, as películas devem apresentar baixa permeabilidade $\mathrm{a}_{2}$. Frutas e hortaliças frescas requerem películas que permitam transferência moderada de gases para reduzir (mas não inibir) a respiração e evitar processos fermentativos resultantes de anaerobiose (DEBEAUFORT e VOILLEY, 1994).

As películas podem ser obtidas de diferentes tipos de materiais, sendo mais utilizados os polissacarídios, as proteínas e os lipídios. As propriedades mecânicas dos filmes à base de proteínas, geralmente, são superiores às dos demais (CHEN, 1995). Os polissacarídios apresentam boas propriedades de formação de filmes e boa barreira aos gases, porém, sendo hidrofílicos, não proporcionam boa barreira à umidade (KESTER e FENNEMA, 1986). Os lipídios oferecem excelente barreira à umidade, mas apresentam problemas relativos à estabilidade oxidativa (CUQ et al., 1995). Devido às vantagens e limitações de cada categoria de componentes dos filmes e coberturas, muitos trabalhos têm envolvido o uso de combinações desses materiais para melhorar as propriedades das coberturas. Películas compostas de polissacarídios e lipídios, por exemplo, combinam as propriedades 
mecânicas e barreira aos gases conferidas pelos polissacarídios com barreira à umidade proporcionada pelos lipídios (KESTER e FENNEMA, 1989; DONHOWE e FENNEMA, 1992; SAPRU e LABUZA, 1994; CHEN E NUSSINOVITCH, 2001).

\section{VANTAGENS POTENCIAIS DA APLICAÇÃO DE PELÍCULAS EM FRUTAS CONSERVADAS POR TMC}

A utilização de películas comestíveis tem sido bastante explorada para revestimento de frutas e hortaliças frescas, visando minimizar a perda de umidade e reduzir as taxas de respiração, além de conferir aparência brilhante e atraente (BALDWIN et al., 1999; HENRIQUE e CEREDA, 1999; DIAB et al., 2001; JIANG e LI, 2001). O uso de películas com esse propósito constitui vantagem econômica, evitando a necessidade de estocagem em atmosfera controlada que implicaria em custos operacionais e de equipamento (KESTER e FENNEMA, 1986).

Embora LEISTNER e GORRIS (1995) tenham mencionado a utilização de películas comestíveis como obstáculo ao crescimento microbiano, podendo ser inserida na TMC, o uso de películas comestíveis em frutas conservadas por métodos combinados tem sido até o momento pouco documentado. A adição de película ao final do processo, após a aplicação dos demais métodos, protege as frutas contra alterações indesejáveis durante a estocagem, além de melhorar sua integridade estrutural. A película pode, alternativamente, ser aplicada antes da desidratação osmótica (método geralmente utilizado em TMC para redução da atividade de água), com o objetivo adicional de modificar as transferências de massa durante o processo de desidratação.

\subsection{REDUÇÃO DA PERDA DE UMIDADE DURANTE A ESTOCAGEM}

Uma das principais características das frutas obtidas por TMC é seu alto teor de umidade, que as torna mais semelhantes aos produtos frescos em termos de textura. Dessa forma, é importante evitar a perda de umidade desses produtos. Graças à sua baixa polaridade, as ceras são bastante adequadas como barreira ao transporte de umidade, 
reduzindo a perda de água durante a estocagem (KESTER e FENNEMA, 1986; HAGENMAIER e SHAW, 1992).

BALDWIN et al. (1999) avaliaram os efeitos de dois tipos de cobertura em mangas in natura, à base de celulose e de cera de carnaúba. Ambas reduziram a perda de umidade, especialmente a de cera de carnaúba. HERSHKO e NUSSINOVITCH (1998) observaram que a adição de cobertura à base de alginato reduziu a perda de umidade de alho in natura durante o período de estocagem. SENESI e BIGNARDI (2000) registraram que a adição de película à base de purê de maçã, em pedaços de maçã desidratados reduziu a perda de peso durante a estocagem e melhorou a aparência do produto.

Entre os fatores que reduzem a taxa de permeabilidade ao vapor de água (TPVA) de películas comestíveis, melhorando sua barreira à umidade, destacam-se o aumento de espessura das películas (SOBRAL, 2000) e a adição de compostos lipídicos (GARCIA et al., 2000; AYRANCI e TUNC, 2001; COMA et al., 2001; DIAB et al., 2001). Por outro lado, os plastificantes, aditivos utilizados para melhorar a flexibilidade de filmes, comprometem a barreira à umidade (ARVANITOYANNIS et al., 1998; TANADA-PALMU et al., 2000; DIAB et al., 2001; SOBRAL et al., 2001).

\subsection{AUMENTO NA RELAÇÃO DE PERFORMANCE DURANTE A DESIDRATAÇÃO OSMÓTICA}

A aplicação da TMC em frutas, geralmente envolve redução da atividade de água por meio de desidratação osmótica, que resulta em menores alterações de sabor e textura dos alimentos quando comparada com métodos convencionais de secagem. $O$ inconveniente da desidratação osmótica, quando se busca obter produtos semelhantes aos frescos, é o ganho de solutos. Para reduzir o fluxo de solutos e ao mesmo tempo permitir a perda de água pela fruta, CAMIRAND et al. (1992) sugeriram o revestimento de frutas com película comestível de alta polaridade antes da desidratação osmótica. A classe de filme mais adequada a esse tipo de aplicação é a dos polissacarídios (KESTER e FENNEMA, 1986). Entre esses estão os ésteres de celulose, que apresentam excelentes propriedades de formação de filmes e eficiente barreira ao oxigênio (PARK et al., 1993), e os alginatos e pectinas de 
baixa metoxilação, que formam filmes bastante fortes e íntegros a partir de ligações cruzadas com íons $\mathrm{Ca}^{+2}$ (KESTER e FENNEMA, 1986).

CAMIRAND et al. (1992) definiram o conceito de relação de performance do processo de desidratação osmótica como sendo a relação entre a perda percentual de umidade e o ganho percentual de sólidos durante o processo. Alguns autores registraram aumento na relação de performance como resultado da adição de películas comestíveis antes do processo (CAMIRAND et al., 1992; WONG et al., 1994; LENART e PIOTROWSKI, 2001). AZEREDO e JARDINE (2000) testaram a adição de coberturas de alginato e pectina de baixa metoxilação antes da desidratação osmótica de pedaços de abacaxi. Verificaram as seguintes alterações do processo em relação aos pedaços nãorevestidos: a perda de umidade aumentou em mais de $30 \%$, o ganho de sólidos foi reduzido em cerca de $60 \%$ e a relação de performance triplicou.

\subsection{MELHORIA DA APARÊNCIA}

As películas, especialmente as de baixa permeabilidade a gases, como os polissacarídios, reduzem as taxas de escurecimento enzimático. O escurecimento enzimático consiste em diversas reações que ocorrem a partir de oxidação enzimática de compostos fenólicos pela ação de polifenoloxidases (PPO). A reação acontece como resultado de injúrias mecânicas pós-colheita e da desintegração durante o processamento, que permitem o acesso do $\mathrm{O}_{2}$ aos tecidos e o contato da enzima com o substrato. A principal conseqüência é a formação de melaninas, pigmentos escuros que prejudicam a aceitação de muitas frutas. Já que o $\mathrm{O}_{2}$ é requerido para iniciar a reação, a utilização de filmes comestíveis pode ser útil para reduzir as taxas de escurecimento (MARTINEZ e WHITAKER, 1995). De fato, alguns autores obtiveram redução nas taxas de escurecimento enzimático em frutas como resultado da adição de coberturas à base de polissacarídios (HOWARD e DEWI, 1995; ZHANG e QUANTICK, 1997; JIANG e LI, 2001).

Além da redução das taxas de escurecimento enzimático, outros efeitos importantes são obtidos na aparência de frutas processadas. O brilho e melhor integridade estrutural conferidos pelas películas 
comestíveis tornam os produtos mais atraentes para o consumidor (KESTER e FENNEMA, 1986; HERSHKO e NUSSINOVITCH, 1998; NUSSINOVITCH, 1998; BALDWIN et al., 1999).

\subsection{REDUÇÃO DO CRESCIMENTO MICROBIANO}

O crescimento microbiano em superfícies é a causa mais comum de deterioração de frutas processadas (TORRES e KAREL, 1985). O uso de películas de baixa permeabilidade a gases, como é o caso de polissacarídios, reduz o acesso do oxigênio aos tecidos, minimizando tais alterações (KESTER e FENNEMA, 1986).

Na tecnologia de métodos combinados, geralmente, são utilizados conservantes químicos como um dos obstáculos ao crescimento de microrganismos. Como a concentração de conservantes deve ser tão baixa quanto possível, é importante que se otimize o uso de tais compostos. Assim, pode-se controlar o crescimento microbiano nas superfícies por meio da incorporação de compostos antimicrobianos a películas comestíveis (CHEN et al., 1999; TORRES, MOTOKI e KAREL, 1985). Segundo TORRES et al. (1985), a aplicação direta de conservantes químicos em superfícies de alimentos sólidos exerce efeito limitado, já que o composto difunde-se rapidamente para o interior do alimento. Por outro lado, a adição de compostos antimicrobianos nas películas comestíveis oferecem a vantagem de manter altas concentrações do composto na superfície do produto, local em que é mais requerido, sem elevar muito sua concentração no interior do alimento (VODJANI e TORRES, 1990). De fato, OZDEMIR e FLOROS (2001) observaram que a difusão de sorbato de potássio em filmes de proteína de soro de leite foi dez vezes menor que em alimentos com umidade intermediária.

Segundo CUQ et al. (1995), a difusão de compostos antimicrobianos por meio de películas comestíveis é influenciada por vários fatores, entre os quais: o tipo de película, as características do alimento $(\mathrm{pH}$, atividade de água), as condições de estocagem (temperatura, tempo) e as características do soluto (polaridade, massa molecular). VODJANI e TORRES (1990) destacaram a diminuição da permeabilidade da película ao ácido sórbico com o aumento da concentração de derivados lipídicos (aumento do comprimento de cadeia carbônica de ácidos 
graxos e presença de ligações duplas).

ZHUANG et al. (1991) registraram efetiva inativação microbiana em superfície de tomates resultante de películas de hidroxipropil metilcelulose (HPMC) adicionadas de etanol. TORRES et al. (1985), assim como TORRES e KAREL (1985), obtiveram retenção eficiente de ácido sórbico em filmes protéicos, mantendo altas concentrações superficiais do composto e aumentando a estabilidade de produtos com umidade intermediária. A adição de nisina em filmes celulósicos (COMA et al., 2001) inibiu o crescimento de bactérias gram-positivas. Entretanto, a adição de ácido esteárico para melhorar a barreira do filme à umidade reduziu a atividade antimicrobiana, o que foi atribuído a interações eletrostáticas entre a nisina (catiônica) e o ácido esteárico (aniônico). KO et al. (2001) também registraram eficaz atividade antimicrobiana de nisina adicionada a filmes à base de proteína de soro de leite, que aumentou com a elevação da concentração de nisina e com a redução do $\mathrm{pH}$.

\subsection{RETENÇÃO DE COMPOSTOS DE AROMA}

O aroma característico de determinado alimento resulta da interação de vários compostos voláteis. Assim, a qualidade do produto torna-se comprometida se houver oxidação de compostos de aroma ou perdas por migração através da embalagem. Portanto, o uso de películas comestíveis com boas propriedades de barreira ao oxigênio e aromas pode aumentar a estabilidade sensorial do alimento.

MILLER e KROCHTA (1997) efetuaram revisão sobre a permeabilidade de filmes comestíveis a $\mathrm{O}_{2}$ e aromas. Observaram de forma geral que as proteínas apresentam menor permeabilidade ao $\mathrm{O}_{2}$ do que os polissacarídios não-iônicos. Tal fato foi atribuído à maior polaridade e linearidade da estrutura protéica, resultando em maior coesão. Quanto aos compostos de aroma, existe grande variação em suas propriedades físicas (massa molecular, polaridade), o que torna difícil qualquer generalização sobre a permeabilidade de filmes comestíveis a aromas. Os mesmos autores mencionaram, ainda, a possibilidade da incorporação de compostos de aroma em películas comestíveis para liberação controlada, compensando as perdas desses compostos durante a estocagem e melhorando o perfil sensorial dos produtos. 


\section{CONSIDERAÇÕES FINAIS}

As películas comestíveis oferecem potencial de aplicações em frutas conservadas por métodos combinados, podendo ser utilizadas para aumentar a estabilidade física, química e microbiológica de tais produtos. Além disso, podem aumentar sua aceitação por meio da melhoria da aparência e retenção de suas propriedades de sabor e textura.

Existe, entretanto, a necessidade de pesquisas futuras envolvendo avaliações mais detalhadas e comparativas sobre as propriedades mecânicas, de barreiras e sensoriais de diferentes materiais comestíveis utilizados para a formação de películas.

\section{Abstract}

\section{EDIBLE FILMS AND COATINGS IN FRUITS PRESERVED BY COMBINED METHODS: APPLICATION POTENTIAL}

A literature review was realized about edible films and their possible applications to improve the preserved fruit quality by combined methods. Reduction of moisture loss during storage, increase of the performance ratio of osmotic dehydration, appearance improving, reduction of microbial growth and retention of aroma compounds are described. The films and coatings, besides enhancing stability of fruit products, can improve their sensory quality.

KEY-WORDS: COMBINED METHODS TECHNOLOGY; FRUIT PRESERVATION; EDIBLE COATINGS.

\section{REFERÊNCIAS}

1 AGUILERA, J.M.; CHIRIFE, J. Combined methods for the preservation of foods in Latin America and the CYTED-D project. Journal of Food Engeneering, v. 22, n. 1-4, p. 433-444, 1994.

2 ALZAMORA, S.M.; TAPIA, M.S.; ARGAÍZ, A.; WELLI, J. Application of combined methods technology in minimally processed fruits. Food Research International, v. 26, n. 2, p. 125-130, 1993.

3 ARVANITOYANNIS, I.; NAKAYAMA, A.; AIBA, S. Edible films made from hydroxypropyl starch and gelatin and plasticized by polyols and water. Carbohydrate Polymers, v. 36, n. 2-3, p. 105-119, 1998. 
carbon dioxide transmissions of cellulose-based edible films. Food Chemistry, v. 72, n. 2, p. 231-236, 2001.

5 AZEREDO, H.M.C.; JARDINE, J.G. Desidratação osmótica de abacaxi aplicada à tecnologia de métodos combinados. Ciência e Tecnologia de Alimentos, v. 20 , n. 1 , p. $74-82,2000$.

BALDWIN, E.A.; BURNS, J.K.; KAZOKAS, W.; BRECHT, J.K.; HAGENMAIER, R.D.; BENDER, R.J.; PESIS, E. Effect of two edible coatings with different permeability characteristics on mango (Mangifera indica L.) ripening during storage. Postharvest Biology and Technology, v. 17, n. 3, p. 215-226, 1999.

7 CAMIRAND, W.; KROCHTA, J.M.; PAVLATH, A.E.; WONG, D.; COLE, M.E. Properties of some edible carbohydrate polymer coatings for potential use in osmotic dehydration. Carbohydrate Polymers, v. 17, n. 1, p. 39-49, 1992.

$8 \mathrm{CHEN}, \mathrm{H}$. Functional properties and applications of edible films made of milk proteins. Journal of Dairy Science, v. 78, n. 11, p. 2563-2583, 1995.

9 CHEN, M.J.; WENG, Y.M.; CHEN, W. Edible coatings as preservative carriers to inhibit yeast on Taiwanese-style fruit preserves. Journal of Food Safety, v. 19, n. 2, p. 89-96, 1999.

10 CHEN, S.; NUSSINOVITCH, A. Permeability and roughness determinations of wax-hydrocolloid coatings, and their limitations in determining citrus fruit overall quality. Food Hydrocolloids, v. 15, n. 2, p. 127-137, 2001.

11 CHIRIFE, J.; FAVETTO, G.J. Some physico-chemical basis of food preservation by combined methods. Food Research International, v. 25, n. 5, p. 389396, 1992.

12 COMA, V.; SEBTI, I.; PARDON, P.; DESCHAMPS, A.; PICHAVANT, F.H. Antimicrobial edible packaging based on cellulosis ethers, fatty acids, and nisin incorporation to inhibit Listeria innocua and Staphylococcus aureus. Journal of Food Protection, v. 64, n. 4, p. 470-475, 2001.

13 CUQ, B.; GONTARD, N.; GUILBERT, S. Edible films and coatings as active layers. In: ROONEY, M.L. (Ed.) Active food packaging. London: Blackie Academic \& Professional, 1995. p. 111-142.

14 DEBEAUFORT, F.; VOILLEY, A. Aroma compound and water vapor permeability of edible films and polymeric packagings. Journal of Agricultural and Food Chemistry, v. 42, n. 12, p. 2871-2875, 1994.

15 DIAB, T.; BILIADERIS, C.G.; GERASOPOULOS, D.; SFAKIOTAKIS, E. Physicochemical properties and application of pullulan edible films and coatings in fruit preservation. Journal of the Science of Food and Agriculture, $v$. 81, n. 10, p. 988-1000, 2001. 
DONHOWE, I.G.; FENNEMA, O.R. The effect of relative humidity gradient on water vapor permeance of lipid and lipid-hydrocolloid bilayer films. Journal of the American Oil Chemists' Society, v. 69, n. 11, p. 1081-1087, 1992.

17 GARCIA, M.A.; MARTINO, M.N.; ZARITZKY, N.E. Lipid addition to improve barrier properties of edible starch-based films and coatings. Journal of Food Science, v. 65, n. 6, p. 941-947, 2000.

HAGENMAIER, R.D.; SHAW, P.E. Gas permeability of fruit coating waxes. Journal of the American Society for Horticultural Science, v. 117, n. 1, p. 105-109, 1992.

HENRIQUE, C.M.; CEREDA, M.P. Utilização de biofilmes na conservação póscolheita de morango (Fragaria Ananassa Duch) cv IAC Campinas. Ciência e Tecnologia de Alimentos, v. 19, n. 2, p. 231-240, 1999.

20 HERSHKO, V.; NUSSINOVITCH, A. Physical properties of alginate-coated onion (Allium cepa) skin. Food Hydrocolloids, v. 12, n. 2, p. 195-202, 1998.

21 HOWARD, L.R.; DEWI, T. Sensory, microbiological and chemical quality of minipeeled carrots as affected by edible coating treatment. Journal of Food Science, v. 60, n. 1, p. 142-144, 1995.

22 JIANG, Y.; LI, Y. Effects of chitosan coating on postharvest life and quality of longan fruit. Food Chemistry, v. 73, n. 2, p. 139-143, 2001.

23 KESTER, J.J.; FENNEMA, O.R. An edible film of lipids and cellulose ethers: barrier properties to moisture vapor transmission and structural evaluation. Journal of Food Science, v. 54, n. 6, p. 1383-1389, 1989.

24 KESTER, J.J.; FENNEMA, O.R. Edible films and coatings: a review. Food Technology, v. 40, n. 12, p. 47-59, 1986.

25 KO, S.; JANES, M.E.; HETTIARACHCHY, N.S.; JOHNSON, M.G. Physical and chemical properties of edible films containing nisin and their action against Listeria monocytogenes. Journal of Food Science, v. 66, n. 7, p. 10061011, 2001.

26 LEISTNER, L. Food preservation by combined methods. Food Research International, v. 25, n. 2, p. 151-158, 1992.

27 LEISTNER, L.; GORRIS, L.G.M. Food preservation by hurdle technology. Trends in Food Science and Technology, v. 6, n. 2, p. 41-46, 1995.

28 LENART, A.; PIOTROWSKI, D. Drying characteristics of osmotically dehydrated fruits coated with semipermeable edible films. Drying Technology, v. 19, n. 5, p. 849-877, 2001.

29 MARTINEZ, M.V.; WHITAKER, J.R. The biochemistry and control of enzymatic browning. Trends in Food Science and Technology, v. 6, n. 6, p. 195-200, 1995. 
30 MILLER, K.S.; KROCHTA, J.M. Oxygen and aroma barrier properties of edible films: a review. Trends in Food Science and Technology, v. 8, n. 7, p. 228237, 1997.

31 NUSSINOVITCH, A. Hydrocolloid coating of foods: a review. Leatherhead Food Industry Journal, v. 1, n. 3, p. 174-188, 1998.

32 OZDEMIR, M.; FLOROS, J.D. Analysis and modeling of potassium sorbate diffusion through edible whey protein films. Journal of Food Engeneering, v. 47, n. 2, p. 149-155, 2001.

33 PARK, H.J.; WELLER, C.L.; VERGANO, P.J.; TESTIN, R.F. Permeability and mechanical properties of cellulose-based edible films. Jornal of Food Science, v. 58, n. 6, p. $1361-1364,1993$.

34 SAPRU, V.; LABUZA, T.P. Dispersed phase concentration effect on water vapor permeability in composite methyl cellulose - stearic acid edible films. Journal of Food Processing and Preservation, v. 18. n. 5, p. 359-368, 1994.

35 SENESI, E.; BIGNARDI, B. Film eduli a base di purea di mela per migliorare la qualita e ampliare le funzioni d'uso di spicchi di mela parzialmente essiccati. Rivista di Frutticoltura e di Ortofloricoltura, v. 62, n. 11, p. 61-66, 2000.

36 SOBRAL, P.J.A. Influência da espessura sobre certas propriedades de biofilmes à base de proteínas miofibrilares. Pesquisa Agropecuária Brasileira, v. 35, n. 6, p. 1251-1259, 2000.

37 SOBRAL, P.J.A.; MENEGALLI, F.C.; HUBINGER, M.D.; ROQUES, M.A. Mechanical, water vapor barrier and thermal properties of gelatin based edible films. Food Hydrocolloids, v. 15, p. 423-432, 2001.

38 TANADA-PALMU, P.; HELEN, H.; HYVONEN, L. Preparation, properties and applications of wheat gluten edible films. Agricultural and Food Science in Finland, v. 9, n. 1, p. 23-25, 2000.

39 TORRES, J.A.; KAREL, M. Microbial stabilization of intermediate food surfaces. III. Effects of surface preservative concentration and surface $\mathrm{pH}$ control on microbial stability of an intermediate moisture cheese analog. Journal of Food Processing and Preservation, v. 9, n. 2, p. 107-119, 1985.

40 TORRES, J.A.; MOTOKI, M.; KAREL, M. Microbial stabilization of intermediate moisture food surfaces. I. Control of surface preservative concentration. Journal of Food Processing and Preservation, v. 9, n. 2, p. 75-92, 1985.

41 VODJANI, F.; TORRES, J.A. Potassium sorbate permeability of methylcellulose and hydroxypropyl methylcellulose coatings: effects of fatty acids. Journal of Food Science, v. 55, n. 3, p. 841-846, 1990.

42 WONG, D.W.S.; CAMIRAND, W.M.; PAVLATH, A.E. Development of edible coatings for minimally processed fruits and vegetables. In: KROCHTA, J.M.; 
BALDWIN, E.A.; NISPEROS-CARRIEDO, M.O. (Ed.) Edible coatings and films to improve food quality. Lancaster: Technomic Publishing, 1994. p. 65-88.

43 ZHANG, D.L.; QUANTICK, P.C. Effect of chitosan coating on enzymatic browning and decay during post-harvest storage of litchi (Litchi chinensis Sonn.) fruit. Postharvest Biology and Technology, v. 12, n. 2, p. 195-202, 1997.

44 ZHUANG, R.; BEUCHAT, L.R.; CHINNAN, M.S.; SHEWFELT, R.L.; HUANG, W. Inactivation of Salmonella montevideo on tomatoes by applying cellulosebased edible films. Journal of Food Protection, v. 59, n. 8, p. 808-812, 1991. 